\title{
SOME REMARKS ON TRANSVERSALLY HARMONIC MAPS
}

\author{
JERZY J. KONDERAK* \\ Died 2005 \\ and ROBERT WOLAK \\ Instutyt Matematyki, Uniwersytet Jagielloński, ul. Reymonta 4, 30059 Kraków, Poland \\ e-mail: robert.wolak@im.uj.edu.pl
}

(Received 13 January, 2006; revised 4 September, 2007; accepted 5 September, 2007)

\begin{abstract}
We consider transversally harmonic foliated maps between two Riemannian manifolds equipped with Riemannian foliations. We give various characterisations of such maps and we study the relation between the properties "harmonic" and "transversally harmonic" for a given map. We also consider these problems for particular classes of manifolds: manifolds with transversally almost Hermitian foliations and Riemannian flows.
\end{abstract}

2000 Mathematics Subject Classification. 53C12, 58E20.

Introduction. In his very influential thesis R. T. Smith characterised equivariant harmonic mappings between $G$-manifolds, $G$ being a compact Lie group. Riemannian manifolds with Riemannian foliations, which we call foliated Riemannian manifolds, are natural generalisations of $G$-manifolds. Smith's characterisation has inspired us to define a new class of 'transversally harmonic' foliated mappings between regular foliated Riemannian manifolds (i.e., the foliation is regular) whose study we have initiated in [24]. We extend R. T. Smith's result to foliated mappings between foliated Riemannian manifolds. Moreover, we do not restrict our attention to regular Riemannian foliations - we study foliated mappings between manifolds with singular Riemannian foliations (SRFs). The leaf space of a singular Riemannian foliation can be a very complicated topological space. Even in a very simple case when all leaves of the foliation are compact, the leaf space is a singular stratified space; cf. [14]. The closures of leaves of a regular Riemannian foliation of a compact manifold form an SRF with all leaves compact. Its leaf space is homeomorphic to the orbit space of an action of a compact Lie group on a compact manifold (cf. [29]), and thus it is an algebraic set and a Riemannian polyhedron; cf. [9]. Its smooth structure can be defined equivalently by smooth functions on the manifold that are constant along the leaves or, as is standard in algebraic geometry, via an embedding into a suitable $\mathbb{R}^{k}$, cf. [3]. Therefore the foliated manifold $(M, \mathcal{F})$ can be considered to be a desingularisation of its leaf space $M / \mathcal{F}$. A foliated mapping defines a smooth mapping between the leaf spaces. Thus any transversally harmonic mapping can be considered a harmonic mapping between the leaf spaces. In certain particular cases our approach coincides with that proposed by J. Eells and B. Fuglede in [9].

In the first section we recall basic results on SRFs as well as some results on harmonic and transversally harmonic maps. The second section is dedicated to the

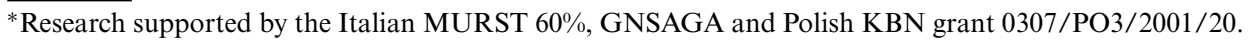


characterisation of foliated harmonic and transversally harmonic mappings between manifolds with Riemannian foliations. At the end of the section we present a theorem on transversally harmonic maps between manifolds foliated by isometric flows. In the subsequent section we study transversally harmonic mappings between Riemannian manifolds foliated by transversally almost Hermitian foliations. Particular attention is paid to taut foliations and the role played by the star operators defined on basic forms. At the end we present some examples.

The authors would like to express their deep gratitude to the referee whose remarks helped improve considerably the paper and in particular, for the remarks concerning the formulation and proof of Proposition 2.1.

\section{Preliminaries.}

1.1. Singular Riemannian foliations. First we recall some useful basic properties of SRFs; cf. [29].

Let $\mathcal{F}$ be an SRF on a compact manifold $M$. The manifold $M$ is stratified by the dimension of leaves; i.e., for any $x \in M$ let us denote by $L_{x}$ the leaf of $\mathcal{F}$ passing through $x$. Then for $r=0, \ldots, n=\operatorname{dim} M$, let $M_{r}=\left\{x \in M: \operatorname{dim} L_{x}=r\right\}$. Obviously, there exist $r_{\min }$ and $r_{\max }$ such that $M_{r}=\emptyset$ for $r<i_{\min }$ or $r>r_{\max } ; r_{\min }$ is the smallest dimension of leaves of the foliation $\mathcal{F}$ and $r_{\max }$ is the greatest dimension of leaves of this foliation. The set $M^{*}=M_{r_{\max }}$ is open and dense in $M$. The set $\Sigma=M-M^{*}$ is a closed subset of $M$ of measure 0 .

In each stratum the foliation $\mathcal{F}$ induces a regular Riemannian foliation, so that $\mathcal{F}^{*}=\mathcal{F} \mid M^{*}$ is a Riemannian foliation of the manifold $M^{*}$.

If the leaves of $\mathcal{F}$ are compact, we can refine our stratification taking into account the holonomy, $[37,19]$, and so on each stratum we have a Riemannian foliation without holonomy. Therefore the foliation $\mathcal{F}^{*}$ on the principal stratum is a Riemannian foliation without holonomy. Hence the natural projection $M^{*} \rightarrow M^{*} / \mathcal{F}^{*}$ is a locally trivial fibre bundle. Using the considerations on basic functions of [37], one easily deduces that any two leaves can be separated by basic functions. Therefore the following lemma is true.

LEMMA 1.1. Let $\left(M_{1}, g_{1}, \mathcal{F}_{1}\right)$ and $\left(M_{2}, g_{2}, \mathcal{F}_{2}\right)$ be two foliated Riemannian manifolds with all leaves compact. Then a mapping $f: M_{1} \rightarrow M_{2}$ is a foliated mapping from $\left(M_{1}, g_{1}, \mathcal{F}_{1}\right)$ to $\left(M_{2}, g_{2}, \mathcal{F}_{2}\right)$ if and only if for any basic function $h$ on $\left(M_{2}, g_{2}, \mathcal{F}_{2}\right)$, hf is a basic function on $\left(M_{1}, g_{1}, \mathcal{F}_{1}\right)$.

On a foliated Riemannian manifold $(M, g, \mathcal{F})$ the $g$-gradient $X_{h}$ of a basic function $h$ is a foliated vector field. Moreover, this vector field is orthogonal to the closures of leaves of $\mathcal{F}$; cf. [37]; also if all leaves are compact, these vector fields span the subbundle orthogonal to the leaves of $\mathcal{F}$ in the principal stratum.

On a manifold $M$ with a foliation $\mathcal{F}$, we can introduce another topology and smooth structure. We take as open subsets of the set $M$ open subsets of leaves. Then the leaves of $\mathcal{F}$ are connected components in this topology and the set $M$ carries a differentiable structure compatible with this topology; we denote this manifold by $M_{\mathcal{F}}$. Moreover, a smooth map $f:\left(M_{1}, \mathcal{F}_{1}\right) \rightarrow\left(M_{2}, \mathcal{F}_{2}\right)$ is foliated if and only if it induces a smooth map $\hat{f}: M_{\mathcal{F}_{1}} \rightarrow M_{\mathcal{F}_{2}}$; cf. [31]. 
1.2. Harmonic and transversally harmonic maps. If $f: M_{1} \rightarrow M_{2}$ is a differentiable map between Riemannian manifolds $\left(M_{1}, g_{1}\right)$ and $\left(M_{2}, g_{2}\right)$, then the differential $d f$ is a section of the bundle $T^{*} M_{1} \otimes f^{-1} T M_{2} \rightarrow M_{1}$, where $f^{-1} T M_{2}$ denotes the pull-back bundle of $T M_{2}$ by the map $f$. The bundle $T^{*} M_{1} \otimes f^{-1} T M_{2} \rightarrow$ $M_{1}$ carries the connection $\nabla$ induced by the Levi-Civita connections on $M_{1}$ and $M_{2}$. Then the map $f$ is said to be harmonic if and only if trace $\nabla d f=0$; the trace is calculated with respect to the Riemannian metric on $M_{1}$. On the other hand, harmonic maps may be defined as critical points of the energy functional. In fact, let $e(f)=\frac{1}{2}\|d f\|^{2}$ be the smooth function on $M_{1}$ which to any point $x$ of $M_{1}$ assigns one half the square of the Hilbert-Schmidt norm of the differential $d_{x} f$ of $f$ at that point. The function $e(f)$ is called the energy density and it is a Lagrangian which arises naturally in the Riemannian geometry; cf. [23]. If $M_{1}$ is compact, then the energy $E(f)$ of $f$ is defined as

$$
E(f):=\int_{M_{1}} e(f) d v_{g_{1}},
$$

where $d v_{g_{1}}$ is the measure determined by the Riemannian metric $g_{1}$ on $M_{1}$. Then $f$ is harmonic if and only if it is a critical point of the energy functional. If $M_{1}$ is not compact, then a harmonic map is a critical point of the energy with respect to the compactly supported variations. The map trace $\nabla d f$ is called the tension field of $f$ and usually is denoted by $\tau(f)$. The equation trace $\nabla d f=0$ is the Euler-Lagrange equation of the variational problem defined by the energy functional. For details about harmonic maps see $[13,10,11,12,1]$.

In our paper [24], we generalise the notion of a harmonic map to foliated maps between manifolds with Riemannian foliations. The definition of a transversally harmonic map arises naturally in the context of the transverse geometry of foliations.

Let $\left(M_{1}, \mathcal{F}_{1}, g_{1}\right)$ and $\left(M_{2}, \mathcal{F}_{2}, g_{2}\right)$ be two Riemannian manifolds with regular Riemannian foliations. Let $\nabla^{i}$ be the Levi-Civita connections of the respective metrics and $D^{i}$ the induced transverse partial connections on the orthogonal complement bundles $T \mathcal{F}_{i}^{\perp} \rightarrow M_{i}$, where $i=1,2$. Suppose that $f: M_{1} \rightarrow M_{2}$ is a smooth foliated map; it means that $d f\left(T \mathcal{F}_{1}\right) \subset T \mathcal{F}_{2}$. Then there are given natural bundle maps

$$
\mathrm{U}_{i}: T \mathcal{F}_{i}^{\perp} \rightarrow T M_{i}, \quad \Pi_{i}: T M_{i} \rightarrow T \mathcal{F}_{i}^{\perp},
$$

for $i=1,2$, where $\mathrm{U}_{i}$ is the inclusion of $T \mathcal{F}_{i}^{\perp}$ in $T M_{i}$, and $\Pi_{i}$ is the orthogonal projection of $T M_{i}$ onto $T \mathcal{F}_{i}^{\perp}$. The map $f$ defines the pull-back bundle $f^{-1} T \mathcal{F}_{2}^{\perp} \rightarrow M_{1}$. Take the bundle

$$
\left(T \mathcal{F}_{1}^{\perp}\right)^{*} \otimes_{M_{1}} f^{-1} T \mathcal{F}_{2}^{\perp} \rightarrow M_{1}
$$

Since there are partial basic connections $D^{2}$ and $D^{1}$ in $f^{-1}\left(T \mathcal{F}_{2}^{\perp}\right) \rightarrow M_{1}$ and $\left(T \mathcal{F}_{1}^{\perp}\right) \rightarrow$ $M_{1}$, respectively, there is the naturally induced partial basic connection $D$ on the bundle (1.1). Next, we consider the foliated bundle

$$
\left(T \mathcal{F}_{1}^{\perp}\right)^{*} \otimes\left(T \mathcal{F}_{1}^{\perp}\right)^{*} \otimes f^{-1} T \mathcal{F}_{2}^{\perp} \rightarrow M_{1}
$$

We define the transverse second fundamental form of $f$ as $D\left(\Pi_{2} d f \mathrm{U}_{1}\right)$. This is a section of the bundle (1.2). 
DEFINITION 1.2. A map $f$ is said to be transversally harmonic if and only if the trace of the transverse second fundamental form vanishes. This trace is called the transverse tension field of $f$, and it is denoted by $\tau_{b}(f)$.

We suppose that $\mathcal{U}=\left\{U_{i}, \varphi_{i}, g_{i j}\right\}_{I}$ is a cocycle defining the foliation $\mathcal{F}_{1}$, and let us denote by $\mathcal{V}=\left\{V_{\alpha}, \psi_{\alpha}, h_{\alpha \beta}\right\}_{A}$ a cocycle defining the foliation $\mathcal{F}_{2}$ such that, for any $i \in I$, there exists $\alpha(i) \in A$ with $f\left(U_{i}\right) \subset V_{\alpha(i)}$. Let $\bar{U}_{i}=\varphi_{i}\left(U_{i}\right)$ and $\bar{V}_{\alpha}=\psi_{\alpha}\left(V_{\alpha}\right)$. Then the manifold $N_{1}=\coprod \bar{U}_{i}$ is a transverse manifold of the foliation $\mathcal{F}_{1}$, and $N_{2}=\bigsqcup \bar{V}_{\alpha}$ is a transverse manifold of the foliation $\mathcal{F}_{2}$; cf. [35, 36]. Both transverse manifolds are Riemannian with the induced metrics. The transformations $g_{i j}$ generate a pseudogroup $\mathcal{H}_{1}$, which is called the holonomy pseudogroup of $\mathcal{F}_{1}$ associated with the cocycle $\mathcal{U}$. The transformations $h_{\alpha \beta}$ generate a pseudogroup $\mathcal{H}_{2}$, which is called the holonomy pseudogroup of $\mathcal{F}_{2}$ associated with the cocycle $\mathcal{V}$. Both holonomy groups are pseudogroups of local isometries.

On the level of transverse manifolds, the map $f$ induces a smooth map $\bar{f}$ as, for any $i \in I$, the following diagram is commutative.

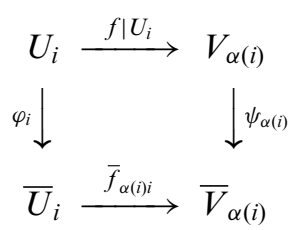

The $\operatorname{map} \bar{f}: N_{1} \rightarrow N_{2}$ is defined as:

$$
\bar{f} \mid \bar{U}_{i}=\bar{f}_{\alpha(i) i}
$$

In [24] we have demonstrated that the mapping $f$ is transversally harmonic if and only if the mapping $\bar{f}$ is harmonic; cf. Theorem 3.1.

A smooth foliated map $f:\left(M_{1}, \mathcal{F}_{1}\right) \rightarrow\left(M_{2}, \mathcal{F}_{2}\right)$ induces a smooth map $\hat{f}: M_{\mathcal{F}_{1}} \rightarrow$ $M_{\mathcal{F}_{2}}$. Let $\tau(\hat{f})$ be the tension field of the map $\hat{f}$ of the manifolds $M_{\mathcal{F}_{1}}$ and $M_{\mathcal{F}_{2}}$ with their naturally induced Riemannian metrics. We say that the mapping $f$ is leaf-wise harmonic if $\tau(\hat{f})=0$; i.e. the mapping $\hat{f}: M_{\mathcal{F}_{1}} \rightarrow M_{\mathcal{F}_{2}}$ is harmonic.

Now suppose that $\mathcal{F}_{1}$ and $\mathcal{F}_{2}$ are Riemannian foliations which are singular and that $f\left(M_{1}^{*}\right) \subset M_{2}^{*}$. Then $f$ is said to be transversally harmonic if the restricted map $\left.f\right|_{M_{1}^{*}}: M_{1}^{*} \rightarrow M_{2}^{*}$ is transversally harmonic.

2. Riemannian foliations. The local geometry of Riemannian foliations is closely related to the geometry of Riemannian submersions. Hence it is natural to expect that results about harmonic maps between the total spaces of Riemannian submersions can be extended to foliated maps between Riemannian foliated manifolds. In this section, we apply the results of Xin on Riemannian submersions, cf. [39], to obtain various characterisations of transversally harmonic maps.

Throughout this section we suppose that there are given two Riemannian foliated manifolds $\left(M_{i}, g_{i}, \mathcal{F}_{i}\right), i=1,2$. We allow foliations to be singular; hence we denote by $M_{i}^{*}, i=1,2$, the union of the leaves of the maximal dimension; cf. Section 1.1. Let $f: M_{1} \rightarrow M_{2}$ be a smooth foliated map such that $f\left(M_{1}^{*}\right) \subset M_{2}^{*}$. If $X$ is a vector on a foliated manifold, then we denote its components the orthogonal to, and tangent to, 
the leaves by $\mathcal{H}(X)$ and $\mathcal{V}(X)$, respectively. By $B_{i}$ we denote the second fundamental form and by $H_{i}$ the mean curvature vector field of leaves of $\mathcal{F}_{i}$ in $\left(M_{i}^{*}, g_{i}\right)$, respectively.

The map $f$ is said to be essentially horizontal if $\left.d f\right|_{T M_{1}^{*}}$ sends $T \mathcal{F}_{1}^{\perp}$ into $T \mathcal{F}_{2}^{\perp}$. The following theorem is a foliated version of Theorem 6.3 of [39]; cf. also [38].

THEOREM 2.1. Let $f: M_{1} \rightarrow M_{2}$ be a smooth foliated mapping between foliated Riemannian manifolds $\left(M_{1}, g_{1}, \mathcal{F}_{1}\right)$ and $\left(M_{2}, g_{2}, \mathcal{F}_{2}\right)$. If $f\left(M_{1}^{*}\right) \subset M_{2}^{*}$ and $f$ is an essentially horizontal map, then $f$ is transversally harmonic if and only if

$$
\mathcal{H}(\tau(f))-\operatorname{trace}_{T \mathcal{F}_{1}} f^{*} B_{2}+f_{*} H_{1}=0 .
$$

Proof. Let $U_{i}$ be open subsets in $M_{i}^{*}$ and $\pi_{i}: U_{i} \rightarrow \bar{U}_{i}$ the submersions defining the foliations $(i=1,2)$ such that $f\left(U_{1}\right) \subset U_{2}$. Then there exists a map $\bar{f}: \bar{U}_{1} \rightarrow \bar{U}_{2}$ such that the following diagram

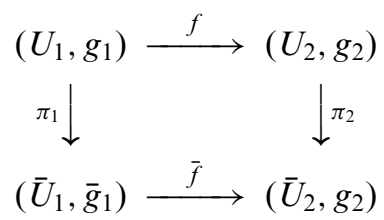

commutes. Here the vertical maps are Riemannian submersions. From [39, equation (6.10)] we have that

$$
\tau(f)=\tau(\bar{f})^{H}+\operatorname{trace}_{T \mathcal{F}_{1}} f^{*} B_{2}-f_{*} H_{1}+\tau(\hat{f}) .
$$

Since $f$ is horizontal, $f^{*} B_{2}$ and $f_{*} H_{1}$ are orthogonal to the leaves of $\mathcal{F}_{2}$. Moreover, $\tau(\hat{f})$ is tangent to the leaves. Hence by taking the horizontal part of both sides of (2.3), we get that $\tau(\bar{f})^{H}=\mathcal{H}(\tau(f))-\operatorname{trace}_{T \mathcal{F}_{1}} f^{*} B_{2}+f_{*} H_{1}$ and hence our assertion follows.

Since there are no minimal SRFs, (cf. [28]), in the following corollary we have to assume that both foliations are regular.

COROLlary 2.1. If both foliations are regular, the foliation $\mathcal{F}_{1}$ is minimal, and $\mathcal{F}_{2}$ totally geodesic, then the map $f$ is harmonic if and only iff is transversally harmonic and leaf-wise harmonic.

Proof. From (2.3) and the assumptions we obtain $\tau(f)^{V}=\tau(\hat{f})$, and so Corollary 2.1 follows.

The theorem below is the foliated version of Theorem 6.4 of [39]; cf. also [38].

THEOREM 2.2. Let $f: M_{1} \rightarrow M_{2}$ be a smooth foliated mapping between regular foliated Riemannian manifolds $\left(M_{1}, g_{1}, \mathcal{F}_{1}\right)$ and $\left(M_{2}, g_{2}, \mathcal{F}_{2}\right)$. If the foliations $\mathcal{F}_{1}$ and $\mathcal{F}_{2}$ are totally geodesic and the horizontal distribution of $\mathcal{F}_{2}$ is integrable, then the map $f$ is transversally harmonic if and only if $\mathcal{H}(\tau(f))=0$. In particular, the harmonicity of $f$ implies its transverse harmonicity.

Proof. We consider again local submersions $\pi_{i}: U_{i} \rightarrow \bar{U}_{i}(i=1,2)$, as in the proof of Theorem 2.1. Let $e_{1}, \ldots, e_{q}$ be an orthonormal frame on $\mathcal{H}\left(T U_{1}\right)$ that is projectable 
via $\pi_{1}$ on $T \bar{U}_{1}$. We apply [39, equation (6.13)] and we get that

$$
\begin{aligned}
\tau(f)= & \tau(\bar{f})^{H}+\tau(\hat{f})+\mathcal{V} \sum_{k=1}^{q} \nabla_{\mathcal{V}\left(f_{*} e_{k}\right)} \mathcal{V}\left(f_{*} e_{k}\right)+\sum_{k=1}^{q} \mathcal{V}\left[\mathcal{H}\left(f_{*} e_{k}\right), \mathcal{V}\left(f_{*} e_{k}\right)\right] \\
& -\mathcal{V}\left(f_{*} \nabla_{e_{k}} e_{k}\right)+2 \mathcal{H} \sum_{k=1}^{q} \nabla_{\mathcal{V}\left(f_{*} e_{k}\right)} \mathcal{H}\left(f_{*} e_{k}\right) .
\end{aligned}
$$

Hence, by taking the horizontal part of the left and right hand sides of the equation above, we get that $\tau(\bar{f})^{H}=\mathcal{H}(\tau(f))-2 \mathcal{H} \sum_{k=1}^{q} \nabla_{\mathcal{V}\left(f_{*} e_{k}\right)} \mathcal{H}\left(f_{*} e_{k}\right)$. Since $\mathcal{H}\left(\nabla_{\mathcal{V}\left(f_{*} X\right)} \mathcal{H}\left(f_{*} Y\right)\right)$ vanishes for any $X, Y$ horizontal projectable vector fields on $U_{1}$, the orthogonal distributions being integrable, (cf. [39, p. 155]), then $f$ is transversally harmonic if and only if $\mathcal{H}(\tau(f))=0$.

We observe that, under the assumptions of Theorem 2.2, the transverse harmonicity does not imply the harmonicity of $f$; cf. [24, Example 4.1].

Apply the Ishihara theorem; cf. [18] and [12, 2.13b]. We get the following characterisation of the transversally harmonic maps.

Proposition 2.1. Suppose that $\mathcal{F}_{i}(i=1,2)$ are minimal foliations and local submersions defining $\mathcal{F}_{2}$ are totally geodesic maps. Then $f$ is transversally harmonic if and only if it sends germs of basic convex functions on $M_{2}$ into the germs of basic subharmonic functions on $M_{1}$.

Proof. The problem is local. Hence we consider open subsets $U_{i} \subset M_{i}(i=1,2)$, as in the proof of Theorem 2.1. We can assume that the submersion $\pi_{1}$ has minimal fibres and $\pi_{2}$ is totally geodesic. Let $h: \bar{U}_{2} \rightarrow \mathbb{R}$ be a smooth map. Then $h$ is convex if and only if $h \pi_{2}$ is basic and convex; cf. [18, Theorem 3.2]; next let $k: \bar{U}_{1} \rightarrow \mathbb{R}$ be a smooth map. Then $k$ is subharmonic if and only if $k \pi_{1}$ is basic and subharmonic, since the laplacian commutes with Riemannian submersions of minimal fibres; e.g. cf. [17]. Then $f$ sends germs of basic convex functions on $M_{2}$ into the germs of basic subharmonic functions on $M_{1}$ if and only if $\bar{f}$ sends germs of convex functions into the germs of subharmonic functions. Thus $\bar{f}$ is harmonic according to Theorem 3.4 of [18], which is equivalent to $f$ being transversally harmonic.

Let us assume that both foliations are compact; i.e., all their leaves are compact. Then the leaf spaces $M_{1} / \mathcal{F}_{1}$ and $M_{2} / \mathcal{F}_{2}$ are orbifolds; cf. [29]. The foliations of both principal strata, $M_{1}^{*}$ and $M_{2}^{*}$, are simple, and the leaf spaces $M_{1}^{*} / \mathcal{F}_{1}$ and $M_{2}^{*} / \mathcal{F}_{2}$ are smooth manifolds that are the principal strata of the corresponding orbifolds $M_{1} / \mathcal{F}_{1}$ and $M_{2} / \mathcal{F}_{2}$, respectively. If $f$ maps $M_{1}^{*}$ into $M_{2}^{*}$, then it induces a smooth mapping $\bar{f}$ of the leaf space $M_{1}^{*} / \mathcal{F}_{1}$ into the leaf space $M_{2}^{*} / \mathcal{F}_{2}$. In this case Theorem 2.1 permits us to formulate the following proposition.

Proposition 2.2. Let $f:\left(M_{1}, g_{1}, \mathcal{F}_{1}\right) \rightarrow\left(M_{2}, g_{2}, \mathcal{F}_{2}\right)$ be a foliated map between two regular foliated Riemannian manifolds that maps $M_{1}^{*}$ into $M_{2}^{*}$. If

(1) the leaves of $\mathcal{F}_{1}$ are compact,

(2) $f$ maps vectors orthogonal to $\mathcal{F}_{1}$ into vectors orthogonal to $\mathcal{F}_{2}$,

(3) the foliation $\mathcal{F}_{1}$ is minimal and the foliation $\mathcal{F}_{2}$ is totally geodesic,

(4) the induced map $\hat{f}: M_{\mathcal{F}_{1}} \rightarrow M_{\mathcal{F}_{2}}$ is harmonic,

then $f$ is harmonic if and only if $\bar{f}$ harmonic. 
Proof. Since the set $M_{1}^{*}$ is open and dense in $M_{1}$, the map $f$ is harmonic if and only if its restriction to $M_{1}^{*}$ is. Therefore the theorem is a simple consequence of Theorem 2.1, in particular formula (2.3), as $f \mid M_{1}^{*}$ is transversally harmonic if and only if the map $\bar{f}$ is harmonic - the foliation $\mathcal{F}_{1}$ on $M_{1}^{*}$ is defined by a global submersion $p_{1}: M_{1}^{*} \rightarrow M_{1}^{*} / \mathcal{F}_{1}$.

Taking into account some results of Section 1.1 we can formulate the following corollary.

Corollary 2.2. Let $f:\left(M_{1}, g_{1}, \mathcal{F}_{1}\right) \rightarrow\left(M_{2}, g_{2}, \mathcal{F}_{2}\right)$ be a foliated diffeomorphism between two foliated Riemannian manifolds. If

(1) the leaves of $\mathcal{F}_{i}, i=1,2$, are compact,

(2) $f$ maps the gradient $X_{h}$ of any basic function $h$ on $\left(M_{1}, g_{1}, \mathcal{F}_{1}\right)$ into the gradient $X_{h f^{-1}}$,

(3) the foliation $\mathcal{F}_{1}$ is minimal and the foliation $\mathcal{F}_{2}$ is totally geodesic,

(4) the induced map $\hat{f}: M_{\mathcal{F}_{1}} \rightarrow M_{\mathcal{F}_{2}}$ is harmonic, then $f$ is harmonic if and only if $\bar{f}$ harmonic.

Proof. As the map $f$ is a foliated diffeomorphism it maps $M_{1}^{*}$ onto $M_{2}^{*}$. The condition (1) coupled with (2) ensures that $f$ maps vectors orthogonal to leaves of $\mathcal{F}_{1}$ on $M_{1}^{*}$ to vectors orthogonal to $\mathcal{F}_{2}$. Hence, the corollary follows from Theorem 2.2.

Let $V: M_{1}^{*} \rightarrow[0,+\infty]$ be the map which to any $x \in M_{1}^{*}$ assigns the volume of the leaf passing through $x$. Then transversally harmonic maps of Riemannian foliated manifolds with compact leaves may be characterised in a variational way as follows.

THEOREM 2.3. Suppose that $\left.\mathcal{F}_{1}\right|_{M_{1}^{*}}$ has only compact leaves. Then $f$ is transversally harmonic if and only iff is a critical point of the functional

$$
\widetilde{E}(f):=\int_{M^{*}}\langle\widetilde{d f}, \widetilde{d f}\rangle \frac{1}{V(x)} d \nu_{g},
$$

where, in formula (2.4), $v_{g}$ is the density on $M_{1}$ defined by the metric $g_{1}, \widetilde{d_{x} f}=p r_{f(x)} \circ$ $d f \circ u_{x}, u_{x}: T_{x} \mathcal{F}_{1}^{\perp} \rightarrow T M_{1}$ is the canonical inclusion, $p r_{f(x)}: T_{f(x)} M_{2} \rightarrow T_{f(x)} \mathcal{F}_{2}^{\perp}$ is the canonical projection.

Proof. According to $\left[\mathbf{8}\right.$, Proposition 4.1] the function $V: M_{1}^{*} \rightarrow \mathbb{R}$ is continuous on an open and dense subset of $M_{1}^{*}$.

Hence we have

$$
\begin{aligned}
\int_{B_{1}^{*}}\langle d \bar{f}, d \bar{f}\rangle d \nu_{\bar{g}} & =\int_{B_{1}^{*}}\langle\bar{f} \bar{f}, d \bar{f}\rangle V(x) \frac{1}{V(x)} d \nu_{\bar{g}} \\
& =\int_{B_{1}^{*}}\left(\langle\overline{d f}, d \bar{f}\rangle \frac{1}{V(x)} \int_{L_{x}} d \mu_{x}\right) d \nu_{\bar{g}} \\
& =\int_{M_{1}^{*}}\langle\tilde{d f}, \tilde{d f}\rangle \frac{1}{V(x)} d \nu_{g},
\end{aligned}
$$

where $L_{x}$ denotes the leaf of $\mathcal{F}_{1}$ passing through $x, M_{1}^{*} / \mathcal{F}_{1}=B_{1}^{*}$, and $d \mu_{x}, d \mu_{g}$ and $d \mu_{\bar{g}}$ are the measures on the leaf $L_{x}, M_{1}^{*}$ and $B_{1}^{*}$ induced by the metric tensors $\left.g_{1}\right|_{L_{x}}$, $g_{1}$ and $\bar{g}_{1}$, respectively. 
Let now $\bar{f}_{t}$ be a variation of $\bar{f}$. Then $\bar{f}_{t}$ may be lifted to the smooth variation $f_{t}: M_{1}^{*} \rightarrow M_{2}^{*}$. Therefore $f$ is transversally harmonic if and only if $\bar{f}$ is a critical point of the energy functional on $\bar{B}_{1}^{*}$. Hence from the equation above we get that

$$
\left.\frac{d}{d t} \int_{B_{1}^{*}}\left\langle d \bar{f}_{t}, d \bar{f}_{t}\right\rangle d \mu_{1}\right|_{t=0}=\left.\frac{d}{d t} \int_{M_{1}^{*}}\left\langle\tilde{d f}_{t}, \tilde{d f}_{t}\right\rangle \frac{1}{V(x)} d v_{g}\right|_{t=0} .
$$

Hence our theorem follows.

2.1. Flows. Let $\mathcal{F}_{1}$ be a Riemannian flow on a compact Riemannian manifold $\left(M_{1}, g_{1}\right)$ and $\mathcal{F}_{2}$ a Riemannian flow on a compact Riemannian manifold $\left(M_{2}, g_{2}\right)$. If the foliations are tangentially oriented, then they are defined by vector fields $\hat{X}_{i}, i=1,2$, respectively. Let $f:\left(M_{1}, g_{1}\right) \rightarrow\left(M_{2}, g_{2}\right)$ be a smooth mapping such that $d f\left(\hat{X}_{1}\right)=\hat{X}_{2}$.

If the top-dimensional basic cohomology group $H^{*}\left(M_{2}, \mathcal{F}_{2}\right)$ is non-trivial, the flow $\mathcal{F}_{2}$ is isometric, or equivalently geodesible; cf. [34, Chapter 6]. Therefore one can normalize $\hat{X}_{2}$ getting a non-singular vector field $X_{2}$ such that there exits a 1-form $\chi_{2}$ for which $\chi_{2}\left(X_{2}\right)=1$ and $i_{X_{2}} d \chi_{2}=0$; cf. [34, Propositions 6.7 and 6.8]. Let $\chi_{1}=f^{*} \chi_{2}$, is a 1-form on $M_{1}$. Let $X_{1}$ be the unique vector field tangent to $\mathcal{F}_{1}$ for which $d f\left(X_{1}\right)=X_{2}$. Then $\chi_{1}\left(X_{1}\right)=1$ and $i_{X_{1}} d \chi_{1}=0$. The subbundles $Q_{1}=\operatorname{ker} \chi_{1}$ and $Q_{2}=\operatorname{ker} \chi_{2}$ are supplementary to $\mathcal{F}_{1}$ and $\mathcal{F}_{2}$, respectively. Next, we modify the Riemannian metric $g_{1}$ putting $\bar{g}_{1}\left(X_{1}, X_{1}\right)=1, \quad \bar{g}_{1}\left(X_{1}, Y\right)=0 \forall Y \in Q_{1}$, and $\bar{g}_{1}(X, Y)=g_{1}(X, Y) \forall X, Y \in$ $Q_{1}$. The Riemannian metric $\bar{g}_{1}$ is bundle-like for $\mathcal{F}_{1}$ and $X_{1}$ is a Killing vector field whose orbits are geodesics; cf. [34, Proposition 6.7]. We make a similar modification $\bar{g}_{2}$. The mapping $f$ is a foliated horizontal mapping of $\left(M_{1}, g_{1}, \mathcal{F}_{1}\right)$ into $\left(M_{2}, g_{2}, \mathcal{F}_{2}\right)$, and both foliations are totally geodesic. Moreover, the induced mapping $\hat{f}: M_{\mathcal{F}_{1}} \rightarrow M_{\mathcal{F}_{2}}$ is an isometry. Therefore the following theorem is a simple consequence of the considerations above and the formula (2.3).

THEOREM 2.4. Let $\mathcal{F}_{1}$ be a tangentially oriented Riemannian flow on a compact Riemannian manifold $\left(M_{1}, g_{1}\right)$ of dimension $m+1$ and $\mathcal{F}_{2}$ a tangentially oriented Riemannian flow on a compact Riemannian manifold $\left(M_{2}, g_{2}\right)$ of dimension $n+1$. If the top-dimensional basic cohomology groups $H^{m}\left(M_{1}, \mathcal{F}_{1}\right)$ and $H^{n}\left(M_{2}, \mathcal{F}_{2}\right)$ are nontrivial and $f$ maps a non-vanishing vector field tangent to $\mathcal{F}_{1}$ to a non-vanishing vector field tangent to $\mathcal{F}_{2}$, then one can modify the Riemannian metrics along the leaves of foliations $\mathcal{F}_{1}$ and $\mathcal{F}_{2}$ in such a way that the mapping $f$ is transversally harmonic if and only if it is harmonic.

COROLlARY 2.3. Let $\mathcal{F}_{1}$ be a tangentially oriented Riemannian flow on a compact Riemannian manifold $\left(M_{1}, g_{1}\right)$ of dimension $m+1$ and $\mathcal{F}_{2}$ a tangentially oriented Riemannian flow on a compact Riemannian manifold $\left(M_{2}, g_{2}\right)$ of dimension $n+1$ such that the top-dimensional basic cohomology groups $H^{m}\left(M_{1}, \mathcal{F}_{1}\right)$ and $H^{n}\left(M_{2}, \mathcal{F}_{2}\right)$ are non-trivial. If there exists a transversally harmonic map which maps a non-vanishing vector field tangent to $\mathcal{F}_{1}$ to a non-vanishing vector field tangent to $\mathcal{F}_{2}$, then there exists a harmonic map between $\left(M_{1}, \bar{g}_{1}\right)$ and $\left(M_{2}, \bar{g}_{2}\right)$ where the Riemannian metrics $\bar{g}_{i}$ are obtained from $g_{i}$ by modifications along the leaves of the flows $\mathcal{F}_{i}, i=1,2$, respectively.

3. Transversally almost Hermitian foliations. In this section we are particularly interested in foliations modelled on some classes of almost Hermitian manifolds that appear in the Gray-Hervella classification; cf. [16]. 
3.1. Transversally almost Hermitian foliations. Let $(M, g, \mathcal{F}, J)$ be a Riemannian manifold $(M, g)$ with foliation $\mathcal{F}$ and an almost complex structure $J$ on the orthogonal subbundle $Q=T \mathcal{F}^{\perp}$. We suppose that $J$ is compatible with $g$; i.e., $g\left(J\left(s_{1}\right), J\left(s_{2}\right)\right)=$ $g\left(s_{1}, s_{2}\right)$ for any $s_{1}, s_{2} \in \Gamma(Q)$. Equivalently, being transversally almost Hermitian means that for any point $x \in M$ there is given a foliation defining submersion $\pi: U_{x} \rightarrow \bar{U}$ of an open neighbourhood $U_{x}$ of $x$ in $M$ on a $2 q$-dimensional almost Hermitian manifold $(\bar{U}, \bar{g}, \bar{J})$ such that $d \pi$ restricted to the normal bundle is an holomorphic isometry; i.e., it preserves the scalar products and commutes with the almost complex structures. Therefore for any cocycle $\mathcal{U}$ defining the foliation $\mathcal{F}$ the transverse manifold $N_{\mathcal{U}}$ has an almost Hermitian structure for which the holonomy pseudogroup $\mathcal{H}_{\mathcal{U}}$ is a pseudogroup of automorphisms; i.e. isometries of the almost Hermitian metric. Likewise any foliation which admits a defining cocycle $\mathcal{U}$ such that the transverse manifold $N_{\mathcal{U}}$ is an almost Hermitian manifold and the holonomy pseudogroup $\mathcal{H}_{\mathcal{U}}$ is a pseudogroup of isometries of this almost Hermitian structure, is transversally almost Hermitian, as then the normal bundle $N(M, \mathcal{F})$ admits a foliated almost Hermitian structure; cf. [35, 36]. The transverse Kähler form on $(M, \mathcal{F})$ is defined in the following way: $F(X, Y):=g^{\perp}\left(X^{\perp}, J\left(Y^{\perp}\right)\right)$ for any $X, Y$ vector fields on $M$, where $X^{\perp}, Y^{\perp}$ are their projections on the subbundle orthogonal to the foliation. If the foliation is transversally almost Hermitian, then the form $F$ is basic. This may be easily seen observing that, locally, with respect to the submersion $\pi_{U}: U \rightarrow \bar{U}$ we have that $F=\pi_{U}^{*} \bar{F}$, where $\bar{F}$ is the Kähler form of the induced almost Hermitian structure $(\bar{U}, \bar{g}, \bar{J})$ on $\bar{U}$.

Let $g$ be any bundle-like metric on the foliated manifold $(M, \mathcal{F})$. Now it induces the volume form $\chi_{\mathcal{F}}$ on the tangent bundle $T \mathcal{F}$ and a basic transverse volume form $\operatorname{vol}_{\mathcal{F}}$. The form $\operatorname{vol}_{\mathcal{F}}$ induces a holonomy invariant volume form $\omega_{\mathcal{F}}$ on the transverse manifold $N$.

Let us also denote by $\kappa$ the mean curvature form of the foliation $\mathcal{F}$. We can modify $\kappa$ without changing the transverse part of the metric so that the 1 -form $\kappa$ is basic; cf. [6]. Using the Masa theorem, (cf. [27]), one proves the following result.

THEOREM 3.1. Let $(M, \mathcal{F})$ be a transversally Hermitian foliation of codimension $q$. If $H^{q}(M, \mathcal{F}) \neq 0$, then there exists a bundle-like metric on $(M, \mathcal{F})$ compatible with the transverse Hermitian structure making all leaves of $\mathcal{F}$ minimal submanifolds; i.e. $\kappa=0$ for that Riemannian metric.

We stress that the modifications to the Riemannian metric do not change the transverse part, so that the foliation remains transversally (almost) Hermitian; see [34, Ch. 7], and [6, p. 260].

3.2. Star operators. The bundle-like metric defines two Hodge star operators:

(i) $*: \Omega^{k}(M) \rightarrow \Omega^{n-k}(M)$,

(ii) the transverse part of the Riemannian metric determines the second Hodge star operator $\star: \Omega^{k}(M, \mathcal{F}) \rightarrow \Omega^{q-k}(M, \mathcal{F})$ acting on basic forms.

This operator corresponds to the Hodge star $\bar{*}$ on holonomy invariant forms on the transverse manifold $N$. The differential operator $d$ restricted to $\Omega^{*}(M, \mathcal{F})$ is denote by $d_{B}$. On the other hand, it is easy to observe that $\star \alpha:=(-1)^{p(q-r)} *\left(\alpha \wedge \chi_{\mathcal{F}}\right)$ for any $\alpha \in \Omega^{r}(M, \mathcal{F})$. 
If $M$ is compact, then on the vector space of basic forms $\Omega^{k}(M, \mathcal{F})$ we have the following natural scalar product: for $\alpha, \beta \in \Omega^{k}(M, \mathcal{F}), k=0, \ldots, q$ define

$$
\langle\alpha, \beta\rangle_{B}=\int_{M} \alpha \wedge \star \beta \wedge \chi_{\mathcal{F}}
$$

One can prove the following result; cf. [20, 21, 34].

THEOREM 3.2. The formal adjoint of $d_{B}$ in $\Omega^{*}(M, \mathcal{F})$ with respect to the scalar product $\langle,\rangle_{B}$ is the operator

$$
\delta_{B}=\left(d_{B}-\kappa \wedge\right)^{\star}: \Omega^{k}(M, \mathcal{F}) \rightarrow \Omega^{k-1}(M, \mathcal{F}),
$$

where $\left(d_{B}-\kappa \wedge\right)^{\star} \beta=(-1)^{q(r+1)+1} \star\left(d_{B}-\kappa \wedge\right) \star \beta$, for any $\beta \in \Omega^{r}(M, \mathcal{F})$.

We can compare $\delta_{B}$ with the standard mapping $\delta=* d *: \Omega^{r}(M) \rightarrow \Omega^{r-1}(M)$. We cannot assume that $\delta$ maps basic forms into basic forms. However, it is not difficult to see that for any $\alpha \in \Omega^{r}(M, \mathcal{F})$, (cf. [34, 7.25],

$$
\delta \alpha=\delta_{B} \alpha+* \gamma(\alpha)
$$

where $* \gamma(\alpha)$ is an $(r-1)$-form orthogonal to $\Omega^{*}(M, \mathcal{F})$ with respect to the standard scalar product in $\Omega(M)$. Therefore, if a basic form $\alpha$ is coclosed on the foliated Riemannian manifold $(M, g, \mathcal{F})$, (i.e. $\delta \alpha=0)$, then it is coclosed in the complex of basic forms (i.e. $\left.\delta_{B} \alpha=0\right)$. Therefore

$$
\delta_{B} \alpha=\star d_{B} \star \alpha-\star(\kappa \wedge \star \alpha)=0 .
$$

If the bundle-like metric is minimal (i.e. $\kappa=0$ ), $\delta_{B} \alpha=\star d_{B} \star \alpha$, and so $\delta_{B} \alpha$ is the lift to $\Omega^{*}(M, \mathcal{F})$ of the formal adjoint $\bar{\delta}$ of the differential $\bar{a}$ in $\Omega^{*}(N)$.

In particular, if $H^{q}(M, \mathcal{F}) \neq 0$, by changing the Riemannian metric along leaves we get that for a coclosed form $\alpha$ in this metric

$$
\delta_{B} \alpha=\star d_{B} \star \alpha=0 .
$$

Therefore the corresponding form $\bar{\alpha}$ on the transverse manifold $N$ is coclosed; i.e. $\bar{*} \bar{d} \bar{\alpha}=0$.

3.3. Special structures. Let $(M, g, \mathcal{F}, J)$ be a foliated transversally almost Hermitian manifold with oriented leaves. Let $F$ be the associated transverse Kähler form. We shall call this foliated manifold transversally cosymplectic if $\delta F=0$.

In the Gray-Hervella classification of almost Hermitian manifolds there is a class of the so called $(1,2)-$ symplectic manifolds. These manifolds are described by the property that the $(1,2)$ component of the differential of the Kähler form vanishes; cf. [16]. In the foliated case, the vanishing of the $(1,2)$ component of the differential of the transverse Kähler form $d F$, considered as a form on $N(M, \mathcal{F})^{c}$, is equivalent to the vanishing of the $(1,2)$ component of the differential of the Kähler form $\bar{F}$ on the transverse manifold. It is so because the pull-back of $\bar{F}$ via the submersions defining the foliated almost Hermitian structures is just the transverse Kähler form $F$. Hence $(M, g, \mathcal{F}, J)$ is transversally $(1,2)$-symplectic if and only if the model manifold is $(1,2)$-symplectic. 
Let $f: M_{1} \rightarrow M_{2}$ be a map between two transversally almost Hermitian manifolds. Then the map $f$ is said to be \pm transversally holomorphic if $d f\left(J_{1}(X)\right)= \pm J_{2} d f(X)$, for all $X \in T \mathcal{F}^{\perp}$. Then as a simple application of the theorem of Lichnerowicz, (see $[26,12,32])$, we obtain the following results.

THEOREM 3.3. Let $\left(M_{k}, g_{k}, \mathcal{F}_{k}, J_{k}\right),(k=1,2)$, be two foliated transversally almost Hermitian manifolds, the foliation $\mathcal{F}_{1}$ being minimal. Suppose that $\left(M_{1}, g_{1}, \mathcal{F}_{1}, J_{1}\right)$ is transversally cosymplectic and $\left(M_{2}, g_{2}, \mathcal{F}_{2}, J_{2}\right)$ is transversally $(1,2)-$ symplectic. Then any foliated map $f: M_{1} \rightarrow M_{2}$ that is transversally (anti) holomorphic is also transversally harmonic.

Proof. The transverse Kähler form $F_{1}$ of $\left(M_{1}, g_{1}, \mathcal{F}_{1}, J_{1}\right)$ is basic and coclosed. According to the considerations of Section 3.2, $\delta_{B} F=0$ as well. Thus the corresponding Kähler form of the transverse manifold is coclosed. Hence the induced mapping between the transverse manifolds is (anti)holomorphic, the domain is cosymplectic, and the target manifold is $(1,2)$-symplectic, so that this mapping is harmonic and therefore the foliated map $f: M_{1} \rightarrow M_{2}$ is transversally harmonic; cf. [24].

REMARK. The Kähler version of the theorem above was proved by E. Barletta and S. Dragomir; cf. [2].

Combining Theorem 3.1 and Theorem 3.3 we obtain the following result.

Corollary 3.1. Let $\left(M_{1}, g_{1}, \mathcal{F}_{1}, J_{1}\right)$ and $\left(M_{2}, g_{2}, \mathcal{F}_{2}, J_{2}\right)$ be two foliated transversally Hermitian manifolds. Suppose that $H^{q_{1}}\left(M_{1}, \mathcal{F}_{1}\right) \neq 0$, codim $\mathcal{F}_{1}=q_{1}$, and the associated transverse Kähler form $F_{1}$ on $\left(M_{1}, \mathcal{F}_{1}\right)$ is coclosed in the bundle-like metric making the foliation $\mathcal{F}_{1}$ minimal and $\mathcal{F}_{2}$ is transversally $(1,2)$ symplectic. Then any foliated map, which is transversally (anti) holomorphic, is transversally harmonic.

4. Examples. First, we construct an interesting example of a transversally harmonic map.

EXAMPLE 4.1. Let $(M, g)$ be a Riemannian manifold. We consider $N=T M$, the tangent bundle to $M$. Let $K: T N \rightarrow N$ be the associated connection map; cf. [7]. We have the canonical projection $\pi: N \rightarrow M$. The manifold $N$ carries the Sasaki metric $h$ given by

$$
h(A, B)=g(K(A), K(B))+g(d \pi(A), d \pi(B)),
$$

for any $A, B$ vectors on $N$. The details about the Levi-Civita connection and curvature properties of the manifold $(N, h)$ may be found in [25]. The projection $\pi:(N, h) \rightarrow$ $(M, g)$ is a Riemannian submersion. Hence $N$ is foliated by the fibres of this bundle; the foliation is clearly Riemannian since it is given by a global Riemannian submersion. Let $\left(M_{1}, g_{1}\right),\left(M_{2}, g_{2}\right)$ be two Riemannian manifolds and $\left(N_{1}, h_{1}\right),\left(N_{2}, h_{2}\right)$ the tangent bundle manifolds with their Sasaki metrics. Let $f: M_{1} \rightarrow M_{2}$ be a smooth map. Then if induces the smooth map $F: N_{1} \rightarrow N_{2}$ such that $F=d f$. We have the following 
commutative diagram.

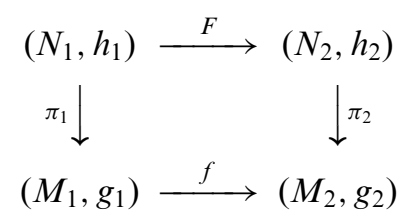

Then the map $f$ is harmonic if and only if $F$ is transversally harmonic. Clearly the map $F$ need not be horizontal. Moreover, the map $F$ is rarely harmonic; cf. [33].

Now let us present some general remarks concerning methods of constructing examples of singular foliations and maps between them.

Let $f:\left(M_{1}, g_{1}, J_{1}, \mathcal{F}_{1}\right) \rightarrow\left(M_{2}, g_{2}, J_{2}, \mathcal{F}_{2}\right)$ be a foliated map between two foliated almost Hermitian manifolds. Then, if the leaf closures of $\mathcal{F}_{1}$ and $\mathcal{F}_{2}, J_{1}$ and $J_{2}$, respectively, are invariant, the almost Hermitian structures are also foliated for the leaf closure foliations; cf. Proposition 2 of [19]. In particular, if the mapping $f$ sends the principal stratum $M_{1}^{*}$ into $M_{2}^{*}$, its restriction to this stratum is transversally holomorphic for the leaf closure foliation. For example, the condition is satisfied if the commuting sheaves $\mathcal{C}_{i}$ of the foliations $\mathcal{F}_{i}$ are $J_{i}$ - invariant, respectively. Now assume that the foliations are transversally Kähler. Then the leaf closure space is a singular Kähler space; cf. [19]. Moreover, from the construction of this structure it is evident that a transversally holomorphic map $f$ that sends the principal stratum $M_{1}^{*}$ into $M_{2}^{*}$ is transversally holomorphic for the leaf closure foliations. The induced mapping $\bar{f}$ on the transverse manifold of the principal stratum is holomorphic for the induced Kähler structures and so, according to Lichnerowicz's Theorem, (cf. [26]), it is harmonic. Thus the mapping $f: M_{1}^{*} \rightarrow M_{2}^{*}$ is transversally harmonic and hence the mapping $f: M_{1} \rightarrow M_{2}$ itself is transversally harmonic.

One of the methods of obtaining transversally holomorphic maps is the suspension construction. The remark above, Theorem 3.3 and the suspension construction can be used to provide examples of transversally harmonic maps in the singular case. See [24] for interesting examples.

First we present the construction of a suspension that can be found in [30] and then use it to construct foliation preserving maps between foliated manifolds.

Let $(F, g)$ be a Riemannian manifold and $\operatorname{Isom}(F, g)$ the group of its isometries. Let us choose any smooth manifold $S$, let $\pi_{1}(S)=G$ be its fundamental group, and $h$ a representation of the group $G$ into $\operatorname{Isom}(F, g)$. Let us take the Cartesian product $\tilde{S} \times F$, where $\tilde{S}$ is the universal covering of $S$. The group $G$ acts on this product via the deck transformations on $\tilde{S}$ and the representation $h$ on $F$ in the following way:

$$
(s, v) \cdot \gamma=(s \cdot \gamma, h(\gamma)(v))
$$

The product $\tilde{S} \times F$ is equipped with the Riemannian metric $g_{s} \times g$, where $g_{s}$ is a Riemannian metric lifted from $S$ to $\tilde{S}$. The action of $G$ on $\tilde{S} \times F$, denoted by the same letter $h$, is isometric for this Riemannian metric. It is a totally discontinuous action and we denote the quotient manifold $\tilde{S} \times F / h$ by $M(S, F ; h)$. It is a fibre bundle over $S$ with the standard fibre $F$. The projection $\tilde{S} \times F \rightarrow F$ defines a foliation of $\tilde{S} \times F$ that is $G$-invariant for the action just defined. It projects to a foliation $\mathcal{F}_{M}$ on $M(S, F ; h)$ that is transverse to the fibres. Its leaves are covering spaces of $S$. In the induced 
Riemannian metric $g_{M}$, the foliation by the fibres is totally geodesic, and the foliation $\mathcal{F}_{M}$ is Riemannian.

Let $\left(S_{1}, g_{S 1}\right),\left(S_{2}, g_{S 2}\right),\left(F_{1}, g_{1}\right)$, and $\left(F_{2}, g_{2}\right)$ be four Riemannian manifolds. Let us denote by $G_{i}$ the fundamental group of the manifold $S_{i}$, and by $h_{i}$ a representation of $G_{i}$ into the group $\operatorname{Isom}\left(F_{i}, g_{i}\right)$ of isometries of the Riemannian manifold $\left(F_{i}, g_{i}\right)$, $(i=1,2)$. Let $f: S_{1} \rightarrow S_{2}$ be a smooth map and $\tilde{f}: \tilde{S}_{1} \rightarrow \tilde{S}_{2}$ its lift to the universal coverings of these manifolds. Then $\tilde{f}$ is $\left(G_{1}, G_{2}\right)$-equivariant. Let us denote by $\pi_{1}(f): G_{1} \rightarrow G_{2}$ the map induced by $f$ on the fundamental groups, and let $h_{i}: G_{i} \rightarrow$ $\operatorname{Isom}\left(F_{i}, g_{i}\right)$ be representations of $G_{i},(i=1,2)$, respectively. Choose a map $\phi: F_{1} \rightarrow F_{2}$ which is $\left(G_{1}, h_{1} ; G_{2}, h_{2}\right)$-equivariant; i.e. $\phi\left(v \cdot h_{1}(\gamma)\right)=\phi(v) . h_{2}\left(\pi_{1}(f)(\gamma)\right)$ for any $v \in F_{1}$ and any $\gamma \in G_{1}$. The map $\tilde{\psi}: \tilde{S}_{1} \times F_{1} \rightarrow \tilde{S}_{2} \times F_{2}$ defined by $\tilde{\psi}=(\tilde{f}, \phi)$ is $\left(G_{1}, G_{2}\right)$ equivariant. Therefore it induces a map $\psi: M_{1}\left(S_{1}, F_{1} ; h_{1}\right) \rightarrow M_{2}\left(S_{2} F_{2} ; h_{2}\right)$. The induced Riemannian metrics on $M_{1}$ and $M_{2}$ we denote by $g_{M 1}$ and $g_{M 2}$, respectively. The following lemmas are fundamental for constructing transversally harmonic maps; cf. [24].

LemMA 4.1. The map $\phi: F_{1} \rightarrow F_{2}$ is harmonic if and only if $\psi$ is transversally harmonic.

Lemma 4.2. The maps $f: S_{1} \rightarrow S_{2}, \phi: F_{1} \rightarrow F_{2}$ are harmonic if and only if the induced suspended map $\psi: M_{1}\left(S_{1}, F_{1} ; h_{1}\right) \rightarrow M_{2}\left(S_{2}, F_{2} ; h_{2}\right)$ is harmonic.

Let the manifolds $\left(F_{1}, g_{1}\right)$, and $\left(F_{2}, g_{2}\right)$ be almost Hermitian (respectively Kähler) for almost complex structures $J_{1}$ and $J_{2}$, respectively, and let the representations $h_{i}$ take values in $\operatorname{Isom}\left(F_{i}, g_{i}, J_{i}\right)$, the group of holomorphic isometries. It is obvious that the almost complex structures $J_{1}$ and $J_{2}$ induce transverse almost complex structures denoted by the same letters $J_{1}$ and $J_{2}$ on $M_{1}$ and $M_{2}$, respectively. Then the foliations are transversally almost holomorphic, respectively transversally Kählerian, and $\phi:\left(F_{1}, J_{1}\right) \rightarrow\left(F_{2}, J_{2}\right)$ is holomorphic if and only if the induced suspended map $\psi:\left(M_{1}\left(S_{1}, F_{1} ; h_{1}\right), \mathcal{F}_{M_{1}}\right) \rightarrow\left(M_{2}\left(S_{2}, F_{2} ; h_{2}\right), \mathcal{F}_{M_{2}}\right)$ is transversally holomorphic for the induced transverse almost complex structures.

If the manifold $F$ is compact, the leaf closure foliation is the following. Take the closure $K$ of $H(G)$ in the group Isom $(F, g, J)$. The natural action of the group $K$ on $F$ defines an SRF $\mathcal{F}_{K}$. The projection onto $M(S, F ; h)$ of the pull-back of $\mathcal{F}_{K}$ to $S \times F$, is the leaf closure foliation. We have the following result.

The mapping $\psi:\left(M_{1}, g_{M_{1}}, \overline{\mathcal{F}}_{M_{1}}\right) \rightarrow\left(M_{2}, g_{M_{2}}, \overline{\mathcal{F}}_{M_{2}}\right)$ is transversally harmonic, (respectively holomorphic) if and only if the map $\phi:\left(F_{1}, \mathcal{F}_{K_{1}}\right) \rightarrow\left(F_{2}, \mathcal{F}_{K_{2}}\right)$ is transversally harmonic (respectively transversally holomorphic).

The right choice of representations in the examples of [24] can provide us with interesting examples.

Let $f:\left(M_{1}, g_{1}\right) \rightarrow\left(M_{2}, g_{2}\right)$ be a smooth map between two Riemannian manifolds. Assume that there exists a smooth $\mathbb{R}^{k}$ isometric action $\phi$ on $\left(M_{1}, g_{1}\right)$ such that the vector fields $v^{*}$ of the associated infinitesimal action are of constant length; i.e. the vector fields $v^{*}(x)=\left.\frac{d}{d t} \phi(t v, x)\right|_{t=0} \in T_{x} M_{1}$ for any $v \in \mathbb{R}^{k}$ and $x \in M_{1}$. Additionally, assume that these vector fields are annihilated by the differential $d f$ of the mapping $f$. In this case, if the mapping $f$ is harmonic, then it is transversally harmonic. It is a consequence of Theorem 2.2, as the foliation of $M_{1}$ is totally geodesic. 
This remark can be applied in several cases.

- Leaf closure foliations of a regular Riemannian foliation $\mathcal{F}$.

The leaf closure foliation $\overline{\mathcal{F}}$ is an SRF whose leaves (the closures of leaves of $\mathcal{F}$ ) are the foliated orbits of the commuting sheaf $\mathcal{C}$; cf. [29]. The commuting sheaf is locally constant. On the principal stratum, both foliations $\mathcal{F}$ and $\overline{\mathcal{F}}$ are regular, and so locally, leaves of $\overline{\mathcal{F}}$ are foliated orbits of a Lie algebra of transverse Killing vector fields; i.e. locally we have the following property.

Let $p:(U, g) \rightarrow(N, h)$ be a Riemannian submersion whose fibres are leaves of $\mathcal{F}$. There exists a Lie algebra $\mathcal{K}$ of Killing vector fields of $(N, h)$ defining a regular foliation on $N$ and such that its inverse image by $p$ is the foliation $\overline{\mathcal{F}}$.

Putting together the remark above and the holonomy pseudogroup characterization of transversally harmonic maps we can formulate the following theorem.

Let $f:\left(M_{1}, g_{1}, \mathcal{F}_{1}\right) \rightarrow\left(M_{2}, g_{2}, \mathcal{F}_{2}\right)$ be a foliated transversally harmonic map between two foliated complete Riemannian manifolds. Assume that the commuting sheaf of the first one is abelian and that the leaves of the second one are closed (compact). Additionally, let the principal stratum be simply connected; then the commuting sheaf is trivial. Therefore if the vector fields of the commuting sheaf have constant length, then the induced mapping $f:\left(M_{1}, g_{1}, \overline{\mathcal{F}}_{1}\right) \rightarrow\left(M_{2}, g_{2}, \mathcal{F}_{2}\right)$ is transversally harmonic.

- The closures of sheets of totally geodesic foliations.

Similar considerations can be applied to the closures of sheets of totally geodesic foliations. In [4], Cairns demonstrated that they form an SRF. One can find interesting examples in $[\mathbf{1 5}, \mathbf{5}]$.

Finally, using the suspension construction we are going to construct transversally Kähler foliations and their transversally holomorphic maps.

EXAMPLE 4.2. Let $(F, g, J)$ be a compact Kähler manifold and $X$ a holomorphic Killing vector field. Then $J X$ is a holomorphic vector field which is rarely a Killing vector field; cf. [22]. Let $G_{X}$ be the Abelian subgroup of $\operatorname{Isom}(F, g, J)$-the group of holomorphic isometries - generated by the flow of the vector field $X$. Any diffeomorphism of the flow of $J X$ commutes with the elements of $G_{X}$ as $[X, J X]=0$; i.e., the elements of $G_{X}$ and $G_{J X}$ commute. In fact, $L_{X} J(Y)=[X, J Y]-J[X, Y]$, for any vector field $Y$. If we take $Y=X$, then $[X, J X]=0$.

Let $h: \mathbb{Z}^{k} \rightarrow G_{X} \subset \operatorname{Isom}(F, g, J)$ be a representation of the group $\mathbb{Z}^{k}=\pi_{1}\left(\mathbb{T}^{k}\right)$. Suspending $h$ we obtain a compact manifold $M\left(\mathbb{T}^{k}, F ; h\right)$ which is a fibre bundle over $\mathbb{T}^{k}$ with the fibre $F$ equipped with a transversally Kähler foliation $\mathcal{F}$ modelled on $(F, g, J)$ and whose leaves are coverings of $\mathbb{T}^{k}$. Any element of the group $G_{J X}$ defines a foliated diffeomorphism of $\left(M\left(\mathbb{T}^{k}, F ; h\right), \mathcal{F}\right)$, that is transversally holomorphic, as elements of the group $G_{J X}$ are holomorphic diffeomorphisms of $(F, g, J)$.

\section{REFERENCES}

1. P. Baird and J. C. Wood, Harmonic morphisms between Riemannian manifolds (Oxford Univesity Press, 2003).

2. E. Barletta and S. Dragomir, On transversally holomorphic maps of Kählerian foliations, Acta Appl. Math. 54 (1998), 121-134.

3. E. Bierstone, The structure of orbit spaces and the singularities of equivariant mappings, Mon. de Mat. 35 (IMPA, Rio de Janeiro, 1980). 
4. G. Cairns, A general description of totally geodesic foliations, Tôhuku Math. J. 38 (1986), 37-55.

5. G. Cairns and E. Ghys, Totally geodesic foliations on 4-manifolds, J. Diff. Geom. 23 (1986), 241-254.

6. D. Dominguez, Finiteness and tenseness theorems for Riemannian foliations, Amer. J. Math. 120 (1998), 1237-1276.

7. P. Dombrowski, On the geometry of the tangent bundles, J. Reine Angew. Math. 210 (1962), 73-88.

8. R. Edwards, K. Millett and D. Sullivan, Foliations with all leaves compact, Topology 16 (1977), 13-32.

9. J. Eells and B. Fuglede, Harmonic maps between Riemannian polyhedra (Cambridge University Press, 2001).

10. J. Eells and L. Lemaire, A report on harmonic maps, Bull. London Math. Soc. 10 (1978), $1-68$.

11. J. Eells and L. Lemaire, Selected topics in harmonic maps, C.B.M.S., Regional Conference Series, 57 (AMS., Providence, R.I. 1983).

12. J. Eells and L. Lemaire, Another report on harmonic maps, Bull. London Math. Soc. 20 (1988), 385-524.

13. J. Eells and J.H. Sampson, Harmonic mappings of Riemannian manifolds, Amer. J. Math. 86 (1964), 109-160.

14. M. Goresky and R. MacPherson, Intersection homology theory, Topology 19 (1980), $135-165$.

15. E. Ghys, Classification des feuilletages totalement géodésiques de codimension un, Comm. Math. Helv. 58 (1983), 543-572.

16. A. Gray and L. Hervella, The sixteen classes of almost Hermitian manifolds and their linear invariants, Annali Mat. Pura Appl. 123 (1980), 35-58.

17. S. Helgason, Differential geometry of symmetric spaces (Academic Press, 1962).

18. T. Ishihara, A mapping of Riemannian manifolds which preserves harmonic functions, J. Math. Kyoto Univ. 19 (1979), 215-229.

19. M. Józefowicz and R. Wolak, A few remarks on the geometry of the space of leaf closures of a Riemannian foliation, in Proceedings of a year in Differential Geometry University of Maryland (Banach Center Publications), Vol. 76 (2007), 395-409.

20. F. Kamber and $\mathrm{Ph}$. Tondeur, Foliations and metrics, Proceedings of a Year in Differential Geometry, University of Maryland (Birkhäuser, 1983), 103-152

21. F. Kamber and Ph. Tondeur, Duality theorems for foliations, Astérisque 116 (1984), $108-116$.

22. S. Kobayashi, Transformation groups in differential geometry (Springer, Verlag, 1972).

23. J.J. Konderak, On natural first order Lagrangians, Bull. London Math. Soc. 23 (1991), $169-174$

24. J. Konderak and R. Wolak, On transversally harmonic maps between manifolds with Riemannian foliations, Quart. J. Math. Oxford Ser.(2) 54 (2003), 335-354.

25. O. Kowalski, Curvature of the Riemannian metric on the tangent bundle of a Riemannian manifold, J. Reine und Angew. Math. 220 (1971), 124-129.

26. A. Lichnerowicz, Applications harmoniques et variétés kähleriennes, Sympos. Math. 3 Bologna (1970), 341-402.

27. X. Masa, Duality and minimality in Riemannian foliations, Comm. Math. Helv. 67 (1992), 17-27.

28. V. Miquel and R. Wolak, Minimal singular Riemannian foliations, CRAS 342 (2006), $33-37$.

29. P. Molino, Riemannian foliations (Birkhäuser, 1988).

30. C. Moore and C. Schochet, Global analysis on foliated spaces, (Springer-Verlag, 1988).

31. M. Mostow, Continuous cohomology of spaces with two topologies, Mem. Amer Math. Soc. 7 (1976), no. 175.

32. J. H. Rawnsley, $f$-structures, $f$-twistor spaces and harmonic maps, in Geometry Seminar

Luigo Bianchi II, 1984, Lecture Notes in Mathematics 1164 (Springer-Verlag, 1985), 85-159

33. A. Sanini, Applicazioni armoniche tra i fibrati tangenti di variet'a riemanniane, Bol. U.M.I. 6 (2A), (1983), 55-63.

34. P. Tondeur, Geometry of foliations (Birkhäuser, 1997). 
35. R. Wolak, Foliated and associated geometric structures on foliated manifolds, Ann. Fac. Sc. Toulouse 10 (1989), 337-360.

36. R. Wolak, Geometric structures on foliated manifolds (Santiago de Compostela, 1989).

37. R. Wolak, Pierrot's theorem for singular Riemannian foliations, Publ. Mat. 38 (1994), 433-439.

38. Y. L. Xin, Riemannian submersion and equivariant harmonic maps, in Proceedings of the Symposium an Differential Geometry in honour of S. Buchin (World Scientific Publ., Singapore, 1993), 272-287.

39. Y.L. Xin, Geometry of harmonic maps (Birkhäuser, 1996). 